\title{
GLTSCR2 Sensitizes Cells to Hypoxic Injury without Involvement of Mitochondrial Apoptotic Cascades
}

\author{
Ji-Hye Yim Yong-Jun Kim Young-Eun Cho Jeong-Hun Ko Su-Mi Kim \\ Jee-Youn Kim Jae-Hoon Park \\ Department of Pathology and Medical Research Center for Bioreaction to Reactive Oxygen Species, \\ College of Medicine, Kyung Hee University, Seoul, Korea
}

Key Words

GLTSCR2 $\cdot$ Hypoxia $\cdot$ Apoptosis $\cdot$ Caspase $\cdot$ Mitochondrion

\begin{abstract}
Objective: We attempted to identify novel genes that induce hypoxic cell death to better understand the molecular mechanisms underlying hypoxia-induced cell death. Through this process the GLTSCR2 gene was found. The purpose of this work was to investigate the role of GLTSCR2 in hypoxic cell death pathways. Methods: This work focuses on an investigation of roles and mechanisms of GLTSCR2 in hypoxic cell death by means of subtractive hybridization, RTPCR, Western blot, immunocytochemistry, cell death assay, transient gene overexpression, and determination of mitochondrial membrane potential. Results: We found that GLTSCR2 was transcriptionally suppressed by hypoxia, and ectopic expression of GLTSCR2 sensitized cells to hypoxic injury. Interestingly, while the majority of hypoxia-inducible pro-death proteins signal through mitochondrion-dependent pathways, GLTSCR2-overexpressed cells underwent apoptosis in a mitochondrion- and caspase-independent manner. Conclusion: Our data categorizes GLTSCR2 as a proapoptotic protein sensitizing cells to hypoxic injury when overexpressed.

Copyright $\odot 2007$ S. Karger AG, Basel
\end{abstract}

\section{Introduction}

Hypoxia is the most common cellular stress and has important pathological implications in many disease processes including cerebral ischemia, myocardial infarction, and chronic degenerative disorders [1]. In response to hypoxia, cells modify their gene expression pattern to adapt to altered metabolic status or to remove irreversibly damaged cells, resulting in either cell survival or death [2]. Although several protein groups including Bcl-2 family and hypoxia-inducible factor-1 (HIF-1)downstream proteins play fundamental roles in this process [3-5], the entire pathway of hypoxic cell death may be too complex to be explained by the known signaling or effector molecules.

A diverse number of cell death pathways are activated by hypoxia, and depending on the cell type or gene expression profile of hypoxia-exposed cells, apoptotic or necrotic cell death can be triggered [6]. Furthermore, even during the apoptotic process, specific caspase-dependent or caspase-independent mechanisms can be activated $[3,7]$. However, in spite of the activation of diverse signaling networks in hypoxia-induced cell death pathways, mitochondria play a central role not only in the generation of ATP but in the regulation of cell death as

\section{KARGER \\ Fax +4161306 1234 \\ E-Mail karger@karger.ch}

www.karger.com
(C) 2007 S. Karger AG, Basel

$1015-2008 / 07 / 0745-0301 \$ 23.50 / 0$

Accessible online at:

www.karger.com/pat
Jae-Hoon Park

Department of Pathology, College of Medicine, Kyung Hee University

\#1, Hoegi-dong, Dongdaemoon-Koo

Seoul 130-701 (Korea)

Tel. +82 2961 0533, Fax +82 2960 2871, E-Mail jhpark@khu.ac.kr 
well $[8,9]$. For example, in response to hypoxia, prodeath molecules such as cytochrome $c$ or apoptosis-inducing factor (AIF) are released from mitochondria into the cytoplasm, where cell death cascades are activated [10].

To better understand the molecular mechanisms underlying hypoxic cell death, we attempted to identify novel genes that mediate hypoxic cell death by subtraction suppression hybridization. Using this method, we identified the glioma tumor suppressor critical region 2 (GLTSCR2) gene, which was initially cloned as a novel gene that interacts with ICP22 and ICP0 of herpes simplex virus 1 [11]. The GLTSCR2 gene is localized in the putative $1.4-\mathrm{Mb}$ tumor suppressive region of chromosome $19 q$, which is frequently altered in various human tumors, including diffuse gliomas [12]. More recently, GLTSCR2 has been reported to directly regulate the phosphorylation and stability of the phosphatase and tensin homolog deleted on chromosome 10 (PTEN) [13]. While these reports suggest that GLTSCR 2 may participate in tumor development, cell proliferation, or differentiation, the function of GLTSCR2 has yet to be characterized.

The purpose of this work was to investigate the role of GLTSCR2 in hypoxic cell death pathways. Our data categorize GLTSCR2 as a member of hypoxia-responsive pro-death genes that induce cell death when overexpressed.

\section{Materials and Methods}

Antibodies and Reagents

Anti-GLTSCR2 polyclonal antibody was produced by immunizing a rabbit with keyhole limpet hemocyanin-conjugated amino acid residues 178-193 (CTRAKPGPQDTVERPF) of human GLTSCR2. The antibody was purified from the immune serum by affinity chromatography. Anti-smac/DIABLO, anti-endonuclease $\mathrm{G}$, anti-Omi/HtrA2, anti-Bax, anti-Bid, anti-BNip3, and anti-Noxa antibodies were purchased from Santa Cruz Biotechnology. Anti-Bad and anti-phospho-Bad antibodies were purchased from Cell Signaling Technology (Beverly, Mass., USA). Anti-AIF, anti-PARP, and anti-cytochrome $c$ antibodies were purchased from Abcam (Cambridge, UK). All other reagents were obtained from Sigma-Aldrich (St. Louis, Mo., USA), unless otherwise specified.

\section{Cell Culture, Hypoxic Condition, Cell Viability, and Cell}

Death Assay

The human A549 alveolar epithelial cell line was obtained from the American Type Culture Collection (Rockville, Md., USA) and cultured in RPMI 1640 medium (Gibco BRL, Carlsbad, Calif., USA) containing 10\% fetal bovine serum under a $5 \% \mathrm{CO}_{2}$ atmosphere at $37^{\circ} \mathrm{C}$. For hypoxic conditions, cells in medium de- gassed with $95 \% \mathrm{~N}_{2}$ and $5 \% \mathrm{CO}_{2}$ were transferred to a hypoxic chamber (Ruskinn, Bridgend, UK) equipped with an air lock and continuously gassed with $2 \% \mathrm{O}_{2}$ and $5 \% \mathrm{CO}_{2}$ with the remainder as nitrogen. Cells stably expressing Bcl-2 (A549/Bcl-2) were created by transfecting A549 cells with Bcl-2 expressing plasmid (pcDNA-Bcl-2) followed by selection with G418. Apoptotic cells exhibiting altered nuclear morphology following $4^{\prime}, 6^{\prime}$-diamidino-2-phenylindole (DAPI) staining were enumerated manually under a fluorescence microscope. Cell viability was determined using alamar blue [14] according to the manufacturer's protocol.

\section{Subtractive Hybridization}

Subtractive hybridization was performed with a PCR-Select cDNA Subtraction Kit (Clontech Laboratories, Mountain View, Calif., USA) according to the manufacturer's protocol, as described previously [15].

Semiquantitative RT-Polymerase Chain Reaction Analysis

One microgram total RNA extracted from normoxia- or hypoxia-stimulated cells was converted to cDNA. The RT-polymerase chain reaction (PCR) exponential phase was determined as between 20 and 36 cycles to allow for semiquantitative comparisons. The PCR regimen for GLTSCR 2 involved a $94^{\circ} \mathrm{C}$, 5-min initial denaturation step, followed by 34 cycles of $94^{\circ} \mathrm{C}$ for $30 \mathrm{~s}, 54^{\circ} \mathrm{C}$ for $45 \mathrm{~s}$, and $72^{\circ} \mathrm{C}$ for $45 \mathrm{~s}$ using a Gene Amp PCR system 9700 (PerkinElmer). The resulting PCR products were separated on $2 \%$ agarose gels and visualized by ethidium bromide staining. For semiquantitative evaluation, densitometric analysis (Bio-Rad Laboratories) was performed.

\section{Plasmid Construction and Transient Transfection}

DNAs encoding full length GLTSCR2 (accession No. NM_ 015710) or its deletion mutants were amplified by PCR from a human kidney cDNA library and cloned into pcDNA3.1/V5 (Invitrogen, Carlsbad, Calif., USA); this construct was then subcloned into a pEGFP vector (Clontech Laboratories). A549 cells $(3 \times$ $105 / 10$-cm culture dish) were transiently transfected with each plasmid using Magnetofection according to the manufacturer's suggested protocol (Chemicell, Berlin, Germany).

\section{Determination of Mitochondrial Membrane Potential}

Transfected A549 cells were cultured under the indicated conditions, at which time MitoTracker Red CMXRos (Molecular Probes) was added to the medium at a final concentration of 400 nM. After $20 \mathrm{~min}$ of incubation, the cells were harvested and washed 3 times with phosphate-buffered saline (PBS). Fluorescence intensity was measured by flow cytometry (FC 500, Beckmann-Coulter).

\section{Western Blotting and Immunocytochemistry}

Western blotting was performed as described previously [16]. For immunocytochemistry, A549 cells were fixed with 4\% paraformaldehyde in PBS and permeabilized with $0.1 \%$ Triton X-100. After blocking with $3 \%$ bovine serum albumin in PBS, the cells were incubated with primary antibody for $1.5 \mathrm{~h}$ at room temperature. Afterwards, the cells were stained with secondary antibody conjugated with fluorescein isothiocyanate or Texas Red and viewed after DAPI staining with a confocal microscope (META 510, Zeiss). 

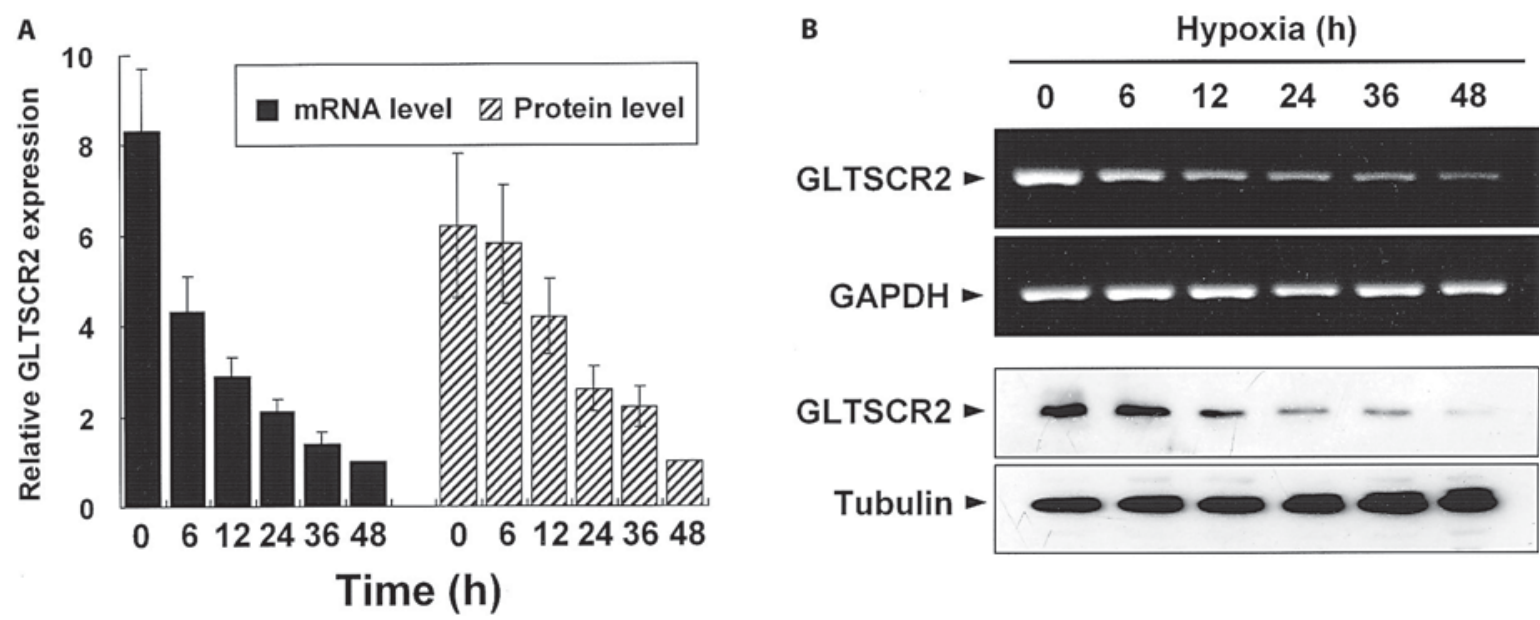

Fig. 1. Hypoxia induces transcriptional downregulation of GLTSCR2 expression. Cells were exposed to hypoxia $\left(2 \% \mathrm{O}_{2}\right)$ for the indicated times. A cDNA was synthesized from total RNA extracted from A549 cells exposed to hypoxia and subjected to RT-PCR analysis, or $30 \mu \mathrm{g}$ of cell lysate extracted from hypoxia-exposed cells were subjected to Western blot analysis using polyclonal anti-GLTSCR2 and anti-tubulin antibodies. Thirty-six and 26 cycles of amplification were performed for GLTSCR2 and GAPDH, respectively. The fold increases of GLTSCR2 expression (mean \pm SD) were determined using densitometry; the GLTSCR2 expression level at $48 \mathrm{~h}$ was arbitrarily defined as 1. B Representative RT-PCR (upper panel) and Western blot (lower panel) results.

Suppression of Endogenous GLTSCR2 Expression by siRNA

Pairs of 19-nucleotide sense and antisense RNA oligomers were independently chemically synthesized and annealed by Bioneer (Daejeon, Korea). The oligonucleotides for GLTSCR2 were as follows: sense, 5'-GCA CAG GCU GCG GGU ACA G-3' and antisense, 5'-CUG UAC CCG CAG CCU GUG C-3', which correspond to human GLTSCR2 coding nucleotides 1455-1475. The selected sequence (siA11) was subjected to a BLAST search to ensure that no other sequence in the human genome other than GLTSCR2 was targeted. The introduction of siRNA was carried out using Lipofectamine 2000 (Invitrogen) according to the manufacturer's suggested protocol.

\section{Results}

\section{Identification of GLTSCR2 as a Hypoxia-Responsive} Gene

Subtractive hybridization was performed to identify hypoxia-responsive genes in A549 lung epithelial cancer cells. Among the 15 putative hypoxia-regulated genes detected by subtractive hybridization, we identified the putative tumor suppressor gene GLTSCR2. To confirm the results of the subtractive hybridization, we performed semiquantitative RT-PCR and Western blot analysis using mRNA and cell lysates extracted from hypoxia-ex- posed A549 cells, respectively. As shown in figure 1A and $\mathrm{B}$, we found a time-dependent decrease of GLTSCR2 transcripts in response to hypoxia. Consistent with the RT-PCR results, protein levels were decreased to approximately $20 \%$ of the levels observed in normoxic cells after $48 \mathrm{~h}$ of hypoxia (fig. 1A, B). Together, our data suggest that hypoxia resulted in transcriptional downregulation of GLTSCR2. Further, the hypoxia-induced downregulation of GLTSCR 2 was observed in a variety of human and mouse cells including endothelial cells, neurons, and neuroblastoma cells (data not shown).

\section{GLTSCR2 Sensitizes Cells to Apoptotic Cell Death \\ Induced by Hypoxia}

The data presented above indicated that hypoxia downregulated the transcription of GLTSCR2. Therefore, we wanted to know whether GLTSCR2 expression levels affected cellular susceptibility to hypoxia. To accomplish this, cells were transiently transfected with pEGFP-GLTSCR2 or a control vector for $12 \mathrm{~h}$ and then exposed to hypoxia $\left(2 \% \mathrm{O}_{2}\right)$ for an additional $24 \mathrm{~h}$. After the hypoxic insult, cell viability was determined by alamar blue. The viability of empty-transfected cells was approximately $94 \%$ after exposure to $2 \%$ hypoxia for $24 \mathrm{~h}$, 


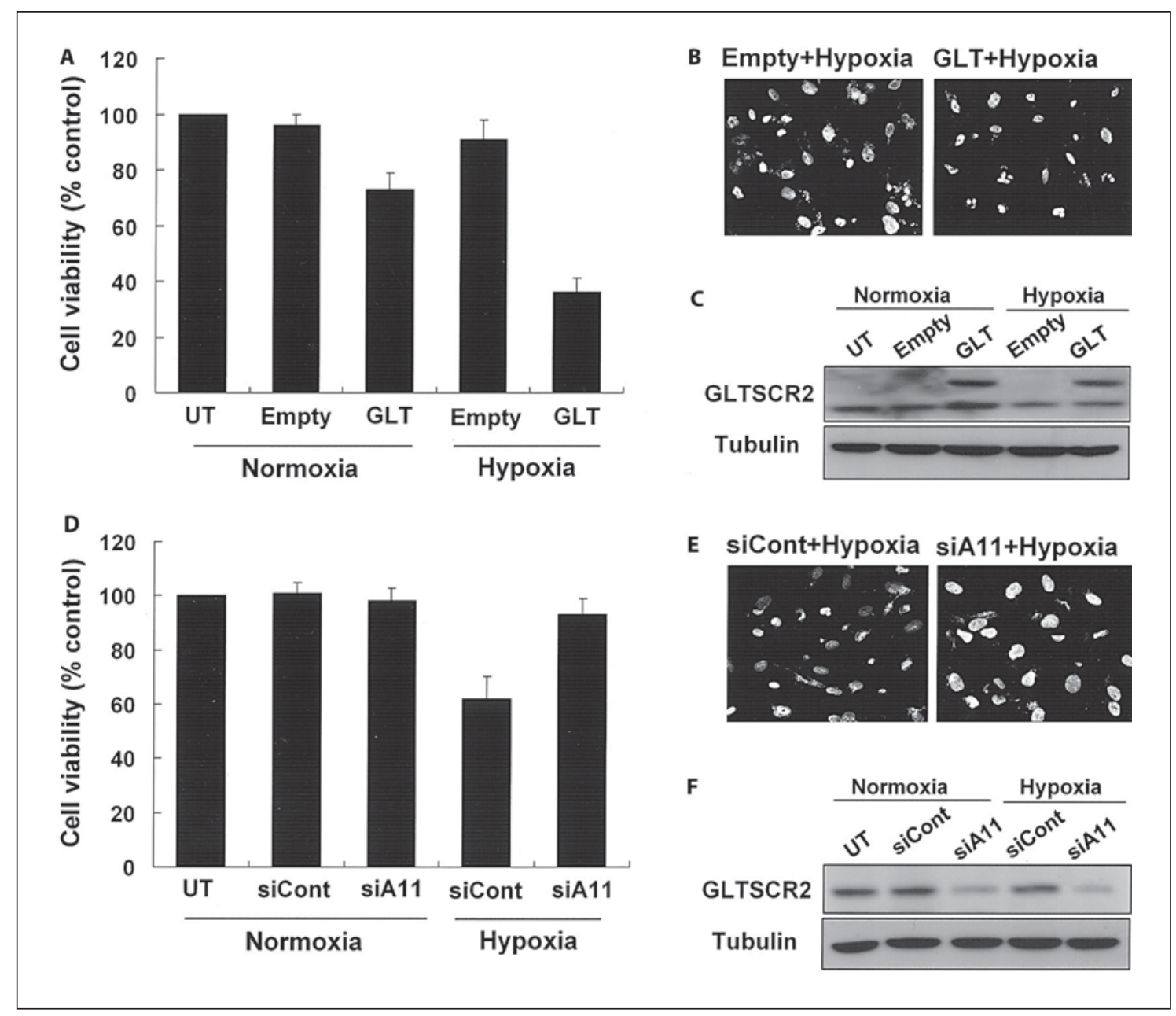

Fig. 2. GLTSCR2 sensitizes cells to hypoxic injury. A A549 cells were transfected with pEGFP-empty or pEGFPGLTSCR2 plasmid and then exposed to normoxia or $2 \%$ hypoxia $12 \mathrm{~h}$ after transfection. Cell viability was determined following $24 \mathrm{~h}$ of hypoxia. B Representative photomicrographs of A after DAPI staining. C Western blots for GLTSCR2 expression level of A. D Cells were transfected with siControl or siA11 for $48 \mathrm{~h}$ and then exposed to normoxia or severe hypoxia $\left(0.2 \% \mathrm{O}_{2}\right)$ for $12 \mathrm{~h}$. Cell viability was determined using alamar blue. Results are presented as the mean \pm SD of three independent experiments. Data represent the percent ratio compared to untreated cells (UT) in normoxic condition. E Representative photomicrographs of $\mathbf{D}$ after DAPI staining. F Western blots for GLTSCR2 expression level of $\mathbf{D}$.

but was decreased to approximately $36 \%$ in cells overexpressing GLTSCR2 (fig. 2A, B). The respective expression level of GLTSCR2 was shown in figure 2C. We subsequently suppressed GLTSCR 2 expression by transfecting GLTSCR2 siRNA (siA11) and exposing cells to severe hypoxia $\left(0.2 \% \mathrm{O}_{2}\right)$ for $12 \mathrm{~h}$. As shown in figure $2 \mathrm{D}$ and $\mathrm{E}$, suppression of GLTSCR2 expression rescued cells from severe hypoxic exposure. Downregulation of GLTSCR2 in siA11-transfected cells was confirmed by Western blots (fig. 2F). These data suggest that GLTSCR2 sensitized cells to hypoxic injury.

\section{Overexpression of GLTSCR2 Does Not Affect the}

Expression Level of Hypoxia-Responsive Proapoptotic

Proteins

Many gene products, including proapoptotic $\mathrm{Bcl}-2$ family proteins, play a role in sensitizing cells to hypoxia to mediate hypoxic cell death [17]. To elucidate whether the sensitization of cells to hypoxic injury by GLTSCR2 overexpression was mediated by induction of hypoxiaresponsive pro-death genes, we investigated the changes of these gene products. A549 cells were transfected with pEGFP-GLTSCR2 and expression of Bax, BNip3, Bid, and 
Fig. 3. GLTSCR2 induces cell death independent of hypoxia-responsive pro-death Bcl-2 family proteins. A A549 cells were transfected with $1 \mu \mathrm{g}$ of pEGFP-GLTSCR2 plasmid and subjected to Western blots for Bax, Bid, BNip3, and Noxa expression after the indicated time intervals. Equalization of the loading was confirmed on the Western blots with tubulin. B Cells were transfected with the indicated plasmid in the presence or absence of $20 \mu \mathrm{M}$ pan-caspase inhibitor $\mathrm{z}$-VAD and cell viability was determined after $48 \mathrm{~h}$ of transfection. Data represent the relative percent ratio compared to empty vector-transfected cells without caspase inhibitor. The means \pm SD of three independent experiments are shown. C A549 cells were transfected with $1 \mu \mathrm{g}$ of pEGFP-GLTSCR2 plasmid and subjected to Western blots for Bad and phospho-Bad (p-Bad) at Ser-136 after the indicated time intervals.

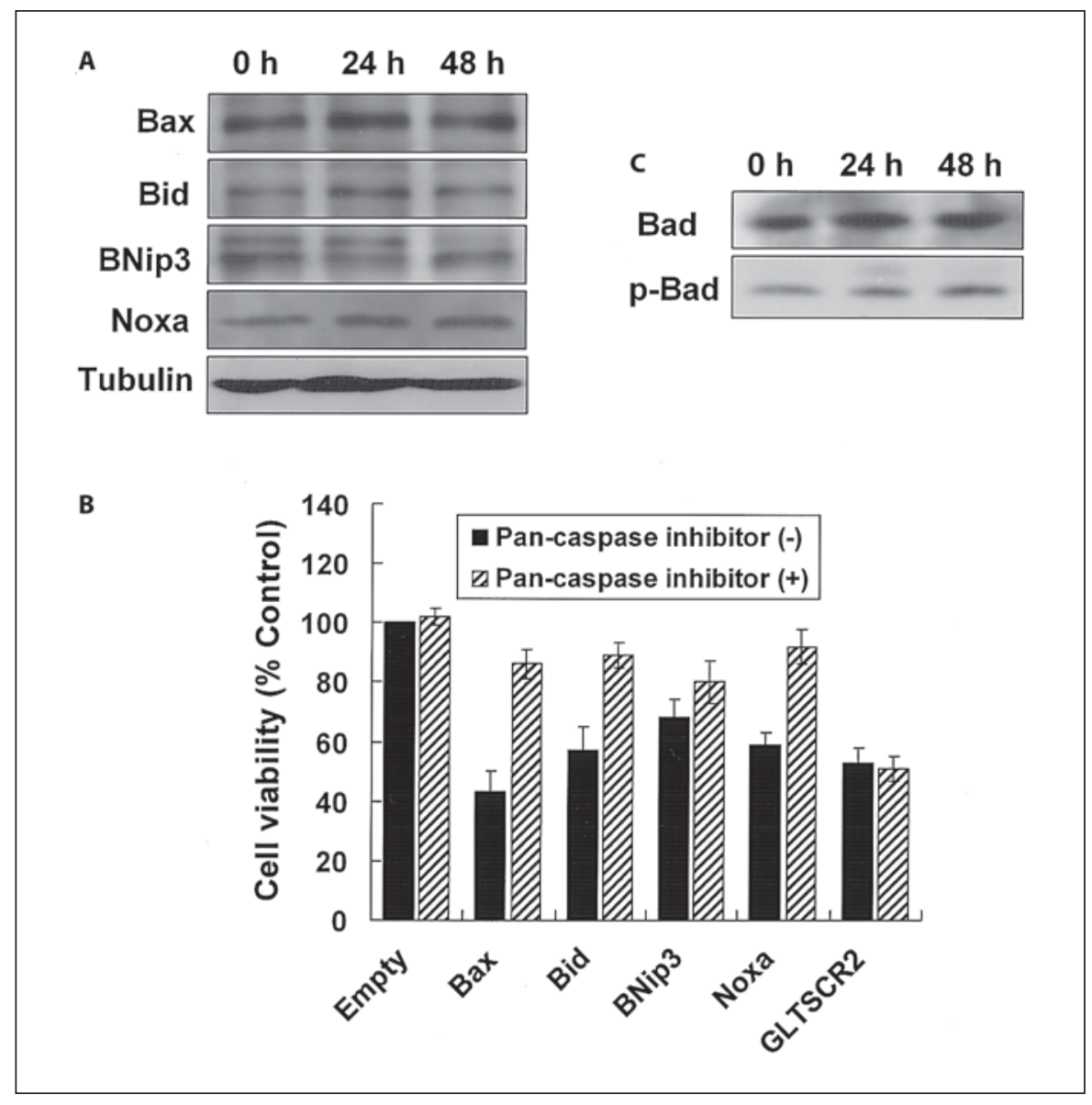

Noxa was determined by Western blotting. As shown in figure $3 \mathrm{~A}$, there was no significant upregulation of these pro-death proteins following $36 \mathrm{~h}$ of GLTSCR2 overexpression. Next, cells were made to overexpress Bax (pcDNA-Bax), Bid (pcDNA-Bid), BNip3 (pcDNABNip3), Noxa (pcDNA-Noxa), or GLTSCR2 (pcDNAGLTSCR2) by transfecting the respective expression vector and then exposing the cells to hypoxia in the presence or absence of pan-caspase inhibitor (z-VAD). Following $24 \mathrm{~h}$ of exposure to hypoxia, cell viability was measured using alamar blue. As shown in figure 3B, the pancaspase inhibitor suppressed hypoxic cell death in Bax-, Bid-, BNip3-, or Noxa-overexpressed cells while it did not affect hypoxic cell death in cells overexpressing GLTSCR2. Together, these data suggest that GLTSCR2 sensitized cells in a Bax-, Bid-, BNip3-, and Noxa-independent manner, and that cell death triggered by GLTSCR2 differs from that of other hypoxia-mediating pro-death proteins. GLTSCR2 is known to stabilize PTEN [13]. Therefore, to investigate the possible involvement of
Bad phosphorylation, A549 cells were transfected with pcDNA-GLTSCR2 and Bad phosphorylation status was determined. As shown in figure 3C, significant alteration in Bad phosphorylation status was not noted.

\section{Sensitization to Hypoxia by GLTSCR2 Is Independent of Mitochondrial Death Cascades}

Mitochondria play a central role in cell death induced by hypoxia. Disruption of the mitochondrial membrane potential (MMP) following the release of pro-death molecules into the cytoplasm is one of the early features of hypoxic cell death [18]. Therefore, we wanted to know whether sensitization to hypoxia by GLTSCR 2 involved mitochondrial apoptotic cascades. To investigate this possibility, cells were transiently transfected with pEGFP-GLTSCR2 or empty vector and the MMP was measured in the GLTSCR2 overexpressing cells with Red CMXRos after 24 and $48 \mathrm{~h}$ posttransfection. As shown in figure $4 \mathrm{~A}$, we did not detect a significant decrease of the MMP following $24 \mathrm{~h}$ of transfection, although a minimal 

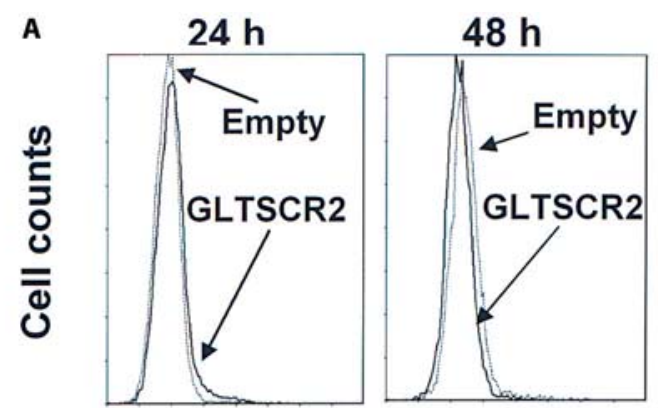

Fluorescence intensity $\rightarrow$

\section{B GLT}
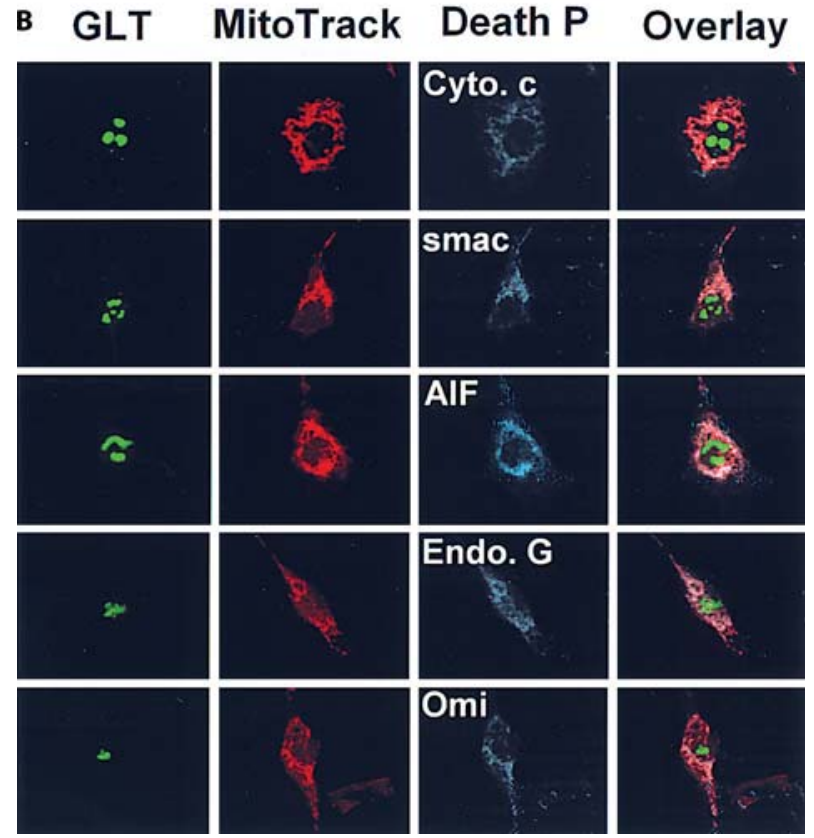

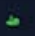
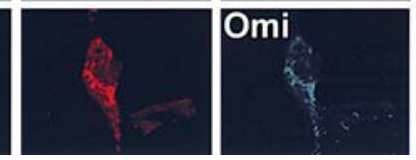

Fig. 4. GLTSCR2 induces cell death independently of the mitochondrial death cascade. A549 cells were transfected with $1 \mu \mathrm{g}$ of empty or pEGFP-GLTSCR2. A Cells were stained with MitoTracker Red CMXRos after the indicated time points. B Cells were double stained with MitoTracker (MitoTrack) and primary antibody for the indicated mitochondrial death protein (Death P), and visualized under a confocal microscope. Representative plots or photographs are shown.

decrease of MMP was noted after $48 \mathrm{~h}$ posttransfection. Next, the release of pro-death proteins from mitochondria, including cytochrome c, AIF, endonuclease G, Omi/ $\mathrm{HtrA}$, and smac/DIABLO, was determined using immunofluorescence staining. Consistent with the insignificant alteration in MMP, a release of pro-death proteins from mitochondria was not noted, further confirming
A
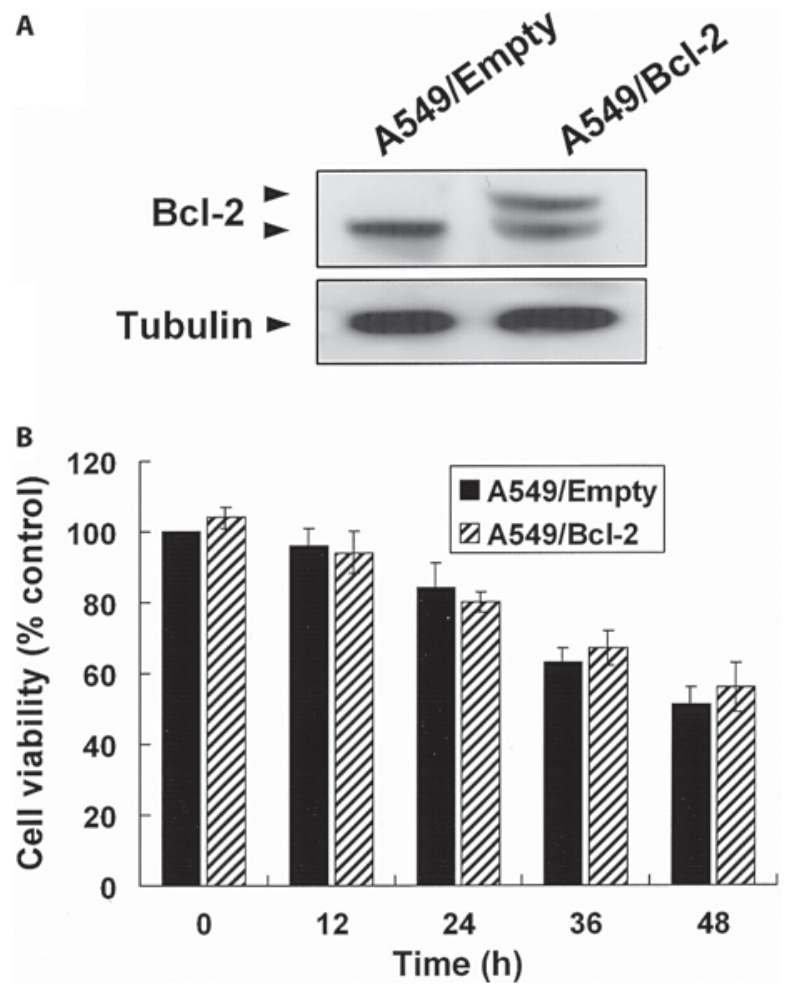

Fig. 5. Cell death induced by overexpression of GLTSCR 2 was not suppressed by co-overexpression of Bcl-2. A A549 cells stably expressing Bcl-2 (A549/Bcl-2) or parent cells (A549/Empty) were lysed and subjected to a Western blot for Bcl-2 or tubulin. B A549/ Bcl-2 or A549/Empty was transfected with $1 \mu \mathrm{g}$ of pEGFPGLTSCR2 and cell viability was determined at the indicated time points. Data represent the relative percent ratio compared to empty vector-transfected cells in A549/Empty cells. Results are presented as the mean \pm SD of three independent experiments.

that GLTSCR2-mediated apoptosis was independent of the mitochondrial apoptotic cascade (fig. 4B).

\section{Effect of Bcl-2 Overexpression on GLTSCR2-Induced}

Cell Death

Bcl-2 regulates various types of cell death pathways by stabilizing mitochondrial membrane permeability [19]. To confirm that GLTSCR2-induced apoptosis does not involve mitochondrial perturbation, we examined whether Bcl-2 could inhibit GLTSCR2-sensitized hypoxic cell death. Cells stably expressing Bcl-2 (A549/Bcl-2) and control cells (A549/Empty) (fig. 5A) were transfected with pEGFP-GLTSCR 2 and exposed to 2\% hypoxia after $12 \mathrm{~h}$ of transfection. The cell death rate was measured after hyp- 
oxic exposure of the indicated time intervals by alamar blue. As depicted in figure $5 \mathrm{~B}, \mathrm{Bcl}-2$ overexpression did not affect the cell death induced by GLTSCR 2 overexpression, indicating that mitochondrial perturbation is not primarily responsible for GLTSCR2-induced cell death.

\section{Discussion}

Hypoxia/ischemia is the most common cause of cell injuries and participates in numerous pathological processes such as ischemic stroke, myocardial infarction and chronic degenerative disorders [20]. When exposed to hypoxia, cells transactivate a variety of genes to adapt to altered metabolic demands or to remove irreversibly damaged cells [21]. Among the transcription factors that respond to hypoxia, HIF-1 $\alpha$ and p53 appear to be key modulators because they transactivate numerous genes responsible for increased oxygen delivery, cell cycle arrest, or apoptosis $[22,23]$. Although survival genes and the molecular pathways that protect against cell death in response to hypoxia are relatively well documented, death-promoting genes induced by hypoxia are less clarified. Here we report the identification of GLTSCR2, a new hypoxia-responding gene that sensitizes cells to hypoxic injury.

Our data obtained from semiquantitative RT-PCR and cell viability assays demonstrated that overexpression of GLTSCR2 sensitized cells to hypoxic injury. However, GLTSCR2 seems to be different from other hypoxia-responding proteins such as BNip3 [3], Noxa [15], and HGTD-P [24] in both their regulation of expression patterns and mechanism of action. First, while a majority of pro-death genes are upregulated by hypoxic stimulation, GLTSCR 2 expression was suppressed by $2 \%$ hypoxia. It is not clear what transcriptional factor(s) induced by hypoxia are responsible for the transcriptional inactivation of the GLTSCR2 promoter. Nevertheless, it might be explained as a cellular adaptation process which protects cells from hypoxic stress. Second, the cell death pathway sensitized by GLTSCR2 was independent of mitochondrial death cascades. Hypoxia-responding pro-death genes, e.g., Bax and Bid [25, 26], perturb mitochondria and result in a loss of MMP and subsequent release of death molecules from the intermembranous space. In the case of GLTSCR2, an activation of such mitochondrial death cascades was not observed. Specifically, (1) membrane potentials were maintained, (2) death molecules from mitochondria were not released, and (3) caspase was not activated. Although these results do not completely rule out the possible involvement of hypoxia-responsive pro-death proteins such as p53 and HIF- $1 \alpha$, they, together with the insignificant alteration in expression of their downstream proteins, strongly imply that GLTSCR2 triggered cell death pathways independent of these proteins. One putative mechanism for GLTSCR2-induced cell death may include PTEN-regulated signaling cascades. However, it is unlikely that the phosphorylation status of Bad by PTEN-Akt cascade is critical for GLTSCR2-induced cell death (fig. 3A). Further studies to elucidate the precise molecular pathway of GLTSCR2 in association with PTEN are ongoing.

Because hypoxia is involved in numerous disease processes such as ischemic stroke, myocardial infarction and chronic degenerative disorders, hypoxia-induced cell death genes are important targets for the development of drugs or other treatment modalities. Overall, the data presented here led us to conclude that GLTSCR2 sensitizes cells to hypoxic injury independent of mitochondria. It may be interesting to study the potential biological significance of GLTSCR2 in transgenic and knockout mice.

\section{Acknowledgment}

This research was supported by the Kyung Hee University Research Fund in 2004 (KHU-20040328).

\section{References}

1 Halterman MW, Federoff HJ: HIF-1 and p53 promote hypoxia-induced delayed neuronal death in models of CNS ischemia. Exp Neurol 1999;159:65-72.

-2 Bunn HF, Poyton RO: Oxygen sensing and molecular adaptation to hypoxia. Physiol Rev 1996;76:839-885.
-3 Saikumar P, Dong Z, Weinberg JM, Venkatachalam MA: Mechanisms of cell death in hypoxia/reoxygenation injury. Oncogene 1998;17:3341-3349.

4 Bruick RK: Expression of the gene encoding the proapoptotic Nip3 protein is induced by hypoxia. Proc Natl Acad Sci USA 2000;97: 9082-9087.

\footnotetext{
5 Greijer AE, van der Wall E: The role of hypoxia inducible factor 1 (HIF-1) in hypoxia induced apoptosis. J Clin Pathol 2004;57: 1009-1014.

6 Brunelle JK, Chandel NS: Oxygen deprivation induced cell death: an update. Apoptosis 2002;7:475-482.
} 
7 Shinzawa K, Tsujimoto Y: PLA2 activity is required for nuclear shrinkage in caspaseindependent cell death. J Cell Biol 2003;163: 1219-1230.

$>8$ Michiels C: Physiological and pathological responses to hypoxia. Am J Pathol 2004;164: 1875-1882.

-9 Malhotra R, Lin Z, Vincenz C, Brosius FC 3rd: Hypoxia induces apoptosis via two independent pathways in Jurkat cells: differential regulation by glucose. Am J Physiol Cell Physiol 2001;281:C1596-C1603.

- 10 Li P, Nijhawan D, Budihardjo I, Srinivasula SM, Ahmad M, Alnemri ES, Wang X: Cytochrome $\mathrm{c}$ and dATP-dependent formation of Apaf-1/caspase-9 complex initiates an apoptotic protease cascade. Cell 1997;91:479489.

-11 Bruni R, Fineschi B, Ogle WO, Roizman B: A novel cellular protein, p60, interacting with both herpes simplex virus 1 regulatory proteins ICP22 and ICP0 is modified in a celltype-specific manner and is recruited to the nucleus after infection. J Virol 1999;73: 3810-3817.

-12 Smith JS, Tachibana I, Pohl U, Lee HK, Thanarajasingam U, Portier BP, Ueki K, Ramaswamy S, Billings SJ, Mohrenweiser HW, Louis DN, Jenkins RB: A transcript map of the chromosome 19q-arm glioma tumor suppressor region. Genomics 2000;64:4450.
13 Okahara F, Ikawa H, Kanaho Y, Maehama T: Regulation of PTEN phosphorylation and stability by a tumor suppressor candidate protein. J Biol Chem 2004;279:4530045303.

14 Nakayama GR, Caton MC, Nova MP, Parandoosh Z: Assessment of the Alamar Blue assay for cellular growth and viability in vitro. J Immunol Methods 1997;204:205-208.

15 Kim JY, Ahn HJ, Ryu JH, Suk K, Park JH BH3-only protein Noxa is a mediator of hypoxic cell death induced by hypoxia-inducible factor 1alpha. J Exp Med 2004;199: 113-124.

16 Kim JY, Yim JH, Cho JH, Kim JH, Ko JH, Kim SM, Park S, Park JH: Adrenomedullin regulates cellular glutathione content via modulation of gamma-glutamate-cysteine ligase catalytic subunit expression. Endocrinology 2006;147:1357-1364.

17 Brunelle JK, Chandel NS: Oxygen deprivation induced cell death: an update. Apoptosis 2002;7:475-482.

18 Armstrong JS: Mitochondria: a target for cancer therapy. Br J Pharmacol 2006;147: 239-248.

19 Breckenridge DG, Xue D: Regulation of mitochondrial membrane permeabilization by BCL-2 family proteins and caspases. Curr Opin Cell Biol 2004;16:647-652.
20 Carmeliet P, Dor Y, Herbert JM, Fukumura D, Brusselmans K, Dewerchin M, Neeman M, Bono F, Abramovitch R, Maxwell P, Koch CJ, Ratcliffe P, Moons L, Jain RK, Collen D, Keshert E: Role of HIF-1alpha in hypoxiamediated apoptosis, cell proliferation and tumour angiogenesis. Nature 1998;394:485490.

$>21$ Bunn HF, Poyton RO: Oxygen sensing and molecular adaptation to hypoxia. Physiol Rev 1996;76:839-885.

22 Moll UM, Zaika A: Nuclear and mitochondrial apoptotic pathways of p53. FEBS Lett 2001;493:65-69.

23 Piret JP, Mottet D, Raes M, Michiels C: Is HIF-1alpha a pro- or an anti-apoptotic protein? Biochem Pharmacol 2002;64:889-892.

24 Lee MJ, Kim JY, Suk K, Park JH: Identification of the hypoxia-inducible factor 1 alpharesponsive HGTD-P gene as a mediator in the mitochondrial apoptotic pathway. $\mathrm{Mol}$ Cell Biol 2004;24:3918-3927.

-25 Hou Q, Hsu YT: Bax translocates from cytosol to mitochondria in cardiac cells during apoptosis: development of a GFP-Bax-stable $\mathrm{H} 9 \mathrm{c} 2$ cell line for apoptosis analysis. Am J Physiol Heart Circ Physiol 2005;289:H477H487.

26 Wei Q, Alam MM, Wang MH, Yu F, Dong Z: Bid activation in kidney cells following ATP depletion in vitro and ischemia in vivo. Am J Physiol Renal Physiol 2004;286:F803F809. 